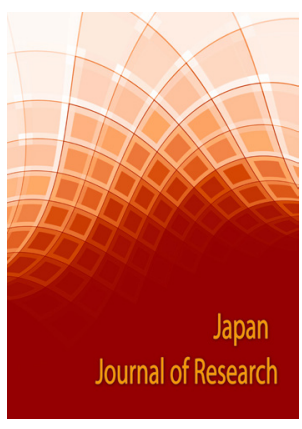

Correspondence

Andrzej Bartke

Department of Internal Medicine, Southern Illinois University School of Medicine, P. O.

Box 19628, Springfield, Illinois, 62794-9628, USA

Email: abartke@siumed.edu

- Received Date: 25 Jan 2020;

- Accepted Date: 03 Feb 2020;

- Publication Date: 13 Feb 2020.

\section{Keywords}

aging; growth hormone; healthspan

\section{Copyright}

(c) 2020 Science Excel. This is an openaccess article distributed under the terms of the Creative Commons Attribution 4.0 International license.

\title{
Growth hormone and aging: focus on healthspan
}

\author{
Andrzej Bartke \\ Department of Internal Medicine, Southern Illinois University School of Medicine, Illinois, USA
}

One of the most effective anti-aging interventions in mice is suppression of growth hormone $(\mathrm{GH})$ signaling. Increased longevity of both females and males was reported in animals in which spontaneous mutations of Pit-1, Prop-1, or Ghrhr genes, or targeted disruption of Ghrh or Ghr produces states of GH deficiency or resistance [1-4]. Importantly, life-prolonging effects of suppressed GH-signaling are not limited to a particular strain, genetic background, or diet, and have been reproduced in independent studies in different laboratories $[4,5]$. Comparably large and consistent extension of longevity is difficult to produce by any means other than severe restriction of caloric intake during most of the lifespan.

Evidence for remarkable "anti-aging" effects of GH deficiency and resistance contrasts with the reports of detrimental effects of the corresponding syndromes in people, including increased cardiovascular risk factors [6,7] and with persistent (although generally poorly supported) claims that $\mathrm{GH}$ therapy can produce various rejuvenating effects in middle-aged and elderly humans. While some individuals with hypopituitarism due to Prop1 mutations reached advanced age [8], isolated deficiency (IGHD) was reported to reduce longevity [9]. Subsequent studies in larger cohorts of individuals with IGHD or GH resistance (Laron syndrome) showed various changes in most common causes of death, but no significant (negative or positive) effects on average longevity $[10,11]$. Intriguingly, a cohort of slightly over 100 individuals with IGHD in northeastern Brazil included one centenarian and one nonagenarian [4]. Possible relationship of GH signaling to late life mortality suggested by these observations appears consistent with relationship of stature to mortality in an unrelated large cohort of normal men [12]. Thus, we must conclude that the association of reduced GH signaling with remarkably extended longevity, which is consistently seen in laboratory stocks of mice is not seen in humans with IGHD or genetic GH resistance. However, there is increasing evidence that reduced GH signaling promotes healthy aging and increases healthspan in both mice and men.
Phenotypic characteristics of mice with various GH-related mutations indicative of the extension of healthspan include reduced incidence and delayed onset of neoplastic disease, reduced oxidative damage to macromolecules, reduced expression of inflammation markers, improved insulin sensitivity and management of blood glucose levels, maintenance of youthful levels of cognitive function into advanced age, reduced immune and collagen aging, and reduced cardiac fibrosis [1-5]. Recent studies provided evidence for additional indications of extended healthspan: improved capacity for DNA repair [13], reduced ovarian aging [14], and younger "biological age" assessed by age-related changes in hepatic DNA methylation $[15,16]$. Future studies should systematically assess time course of agerelated changes in resilience and other measures of healthspan in these long-lived mutants and their normal (wild-type) siblings.

Association of reduced GH action with extension of healthspan in human subjects is suggested by the remarkable protections from age-related disease including cancer and diabetes in the Ecuadorian cohort of Laron dwarfs [10] and protection of Brazilian subjects with IGHD from atherosclerosis and cancers other than skin cancer $[4,11,17]$. Moreover, subjects with IGHD generally experience healthy aging and tend to look younger than their age. Importantly, Dr. Aguiar-Oliveira and his colleagues documented that subjects with IGHD from the cohort they had been following exhibit improved muscle strength, greater resistance to fatigue, reduced incidence of bone fractures, improved insulin sensitivity, and no graying of the hair, while memory, walking, postural balance, and risk of falls are not affected $[4,11,17]$.

What are the implications of these findings? It can be concluded that the anti-aging effects of $\mathrm{GH}$ deficiency or resistance discovered in laboratory studies of mice can be seen also in humans, but the quantitative impact of these syndromes on the aging process is much smaller in our own species than in domesticated mice. Thus, healthspan is extended in both species, but longevity only in mice. What is responsible for this difference? We speculate that this may be due to a different pace-of-life in small rodents 
and large primates. In small rodents, suppression of the somatotropic axis changes characteristics associated with fast paces of life: fast growth, early puberty, high fecundity, and fast aging, to resemble the life course, growth, maturation, and reproductive strategy of species with slower pace-of-life, and, thus, extends longevity. In humans, the "pro-longevity" characteristics of slow pace-of-life are already present, and thus effects of reducing GH actions are more subtle. Obviously, other mechanisms and other characteristics of rodents and primates may also be involved in producing the observed species difference.

Another important implication of these findings is that the normal actions of physiological levels of GH are not optimal for healthy aging, and can be assumed to exert "pro-aging" actions. This may appear counterintuitive, but fits well with the well documented role of other anabolic/nutrient-responsive pathways (insulin/insulin-like growth factors, and mechanistic target of rapamycin) in the control of aging in many organisms, including mammals [18-22].

\section{Conflict of interest}

Andrzej Bartke has no conflict of interest to declare.

\section{References}

1. Bartke A, Sun LY, Longo V. Somatotropic signaling: trade-offs between growth, reproductive development, and longevity. Physiol Rev. 2013;93(2):571-598.

2. Brown-Borg HM. The somatotropic axis and longevity in mice. Am J Physiol Endocrinol Metab. 2015;309(6):E503-E510.

3. Basu R, Qian Y, Kopchick JJ. Mechanisms in endocrinology: Lessons from growth hormone receptor gene-disrupted mice: are there benefits of endocrine defects? Eur J Endocrinol. 2018;178(5):R155-R181.

4. Aguiar-Oliveira MH, Bartke A. Growth Hormone Deficiency: Health and Longevity. Endocr Rev. 2019;40(2):575-601.

5. Bartke A. Single-gene mutations and healthy ageing in mammals. Philos Trans $R$ Soc Lond B Biol Sci. 2011;366(1561):28-34.

6. Rosen T, Bengtsson BA. Premature mortality due to cardiovascular disease in hypopituitarism. Lancet. 1990;336(8710):285-288.

7. Fleseriu M, Hashim IA, Karavitaki N, et al. Hormonal replacement in hypopituitarism in adults: An Endocrine Society Clinical Practice Guideline. J Clin Endocrinol Metab. 2016;101(11):3888-921.

8. Krzisnik C, Grguric S, Cvijovic K, et al. Longevity of the hypopituitary patients from the island Krk: a follow-up study. Pediatr Endocrinol Rev. 2010;7(4):357-62.

9. Besson A, Salemi $S$, Gallati $S$, et al. Reduced longevity in untreated patients with isolated growth hormone deficiency. J Clin Endocrinol Metab. 2003;88(8):3664-7.

10. Guevara-Aguirre J, Balasubramanian P, Guevara-Aguirre M, et al. Growth hormone receptor deficiency is associated with a major reduction in pro-aging signaling cancer, and diabetes in humans. Sci Transl Med. 2011;3(70):70ra13.

11. Aguiar-Oliveira MH, Oliveira FT, Pereira RM, et al. Longevity in untreated congenital growth hormone deficiency due to a homozygous mutation in the GHRH receptor gene. J Clin Endocrinol Metab. 2010;95(2):714-21.

12. He Q, Morris BJ, Grove JS, et al. Shorter men live longer: association of height with longevity and $\mathrm{FOXO} 3$ genotype in American men of Japanese ancestry. PLoS One. 2014;9(5):e94385.

13. Podlutsky A, Valcarcel-Ares MN, Yancey K, et al. The GH/IGF-1 axis in a critical period early in life determines cellular DNA repair capacity by altering transcriptional regulation of DNA repair-related genes: implications for the developmental origins of cancer. Geroscience. 2017;39(2):147-60.

14. Schneider A, Matkovich SJ, Saccon T, et al. Ovarian transcriptome associated with reproductive senescence in the long-living Ames dwarf mice. Mol Cell Endocrinol. 2017:439:328-36.

15. Wang $T$, Tsui $B$, Kreisberg JF, et al. Epigenetic aging signatures in mice livers are slowed by dwarfism, calorie restriction and rapamycin treatment. Genome Biol. 2017;18(1):57.

16. Cole JJ, Robertson NA, Rather MI, et al. Diverse interventions that extend mouse lifespan suppress shared age-associated epigenetic changes at critical gene regulatory regions. Genome Biol. 2017;18(1):58.

17. Costa UM, Oliveira $C R$, Salvatori R, et al. Brazilian adult individuals with untreated isolated GH deficiency do not have accelerated subclinical atherosclerosis. Endocrine connections. 2016;5(1):41-6.

18. Tatar M, Bartke A, Antebi A. The endocrine regulation of aging by insulin-like signals. Science. 2003;299:1346-51.

19. inch CE, Ruvkun $\mathrm{G}$. The genetics of aging. Annual review of genomics and human genetics. 2001;2:435-62.

20. Blagosklonny MV. Rapamycin extends life- and health span because it slows aging Aging (Albany NY). 2013;5(8):592-8.

21. Johnson SC, Rabinovitch PS, Kaeberlein M. mTOR is a key modulator of ageing and age-related disease. Nature. 2013;493(7432):338-45.

22. Kennedy BK, Lamming DW. The mechanistic target of rapamycin: The grand conductor of metabolism and aging. Cell Metab. 2016;23(6):990-1003. 